\title{
Die ,große Lösung“ als gleichheitsrechtliches Gebot - Verfassungsrechtliche Grenzen des dualen Systems der Eingliederungshilfe für Kinder und Jugendliche
}

\author{
Minou Banafsche
}

$\begin{array}{lr}\text { I. Einleitung } & 169\end{array}$

II. Die Vereinbarkeit des „dualen Systems“ mit Art. 3 GG 171

1. Prüfungsmaßstab 171

a) Persönlicher Anwendungsbereich des Art. 5 Abs. 2 UN-BRK 171

b) Sachlicher Anwendungsbereich des Art. 5 Abs. 2 UN-BRK 172

c) Das Verhältnis der UN-BRK zum Grundgesetz 174

d) Ergebnis 176

2. Vereinbarkeit der dualen Eingliederungshilfe mit Art. 3 GG 176

a) Gleichheitsrechtlicher Prüfungsmaßstab 177

b) Rechtlich relevante Ungleichbehandlung 179

aa) Zuständigkeitsdivergenzen $\quad 179$

bb) Leistungsrechtliche Divergenzen $\quad 180$

cc) Ergebnis 181

c) Verfassungsrechtliche Rechtfertigung der Ungleichbehandlung 181

aa) Zuständigkeitsdivergenzen 182

bb) Leistungsrechtliche Divergenzen 183

cc) Ergebnis 185

3. Auflösung der Ungleichbehandlung 185

a) Vorzüge einer einheitlichen Regelung per se 186

b) Vorzüge einer einheitlichen Regelung im SGB XII 186

c) Vorzüge einer einheitlichen Regelung im SGB VIII (,große Lösung“) 187

aa) Die gesetzliche Verortung der Eingliederungshilfe im SGB XII 187

bb) Größere Sachnähe des SGB VIII 190

d) Ergebnis 191

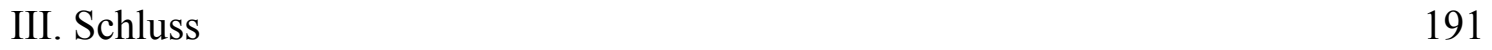

\section{Einleitung}

Das deutsche Sozialleistungsrecht ordnet Kinder und Jugendliche mit Behinderungen unterschiedlichen Regelungssystemen je nach Art ihrer Behinderung zu. Kinder und Jugendliche mit seelischen Behinderungen erhalten Eingliederungshilfe nach $\S 35 \mathrm{a}$ 
SGB VIII, Kinder und Jugendliche mit körperlichen und/oder geistigen Behinderungen erhalten bei Vorliegen aller Leistungsvoraussetzungen Eingliederungshilfe nach den $\S \S 53$ ff. SGB XII.

Unter dem Punkt „Neue Perspektiven für Kinder und Jugendliche mit Behinderung“ postuliert der 13. Kinder- und Jugendbericht Folgendes:

„Die Einnahme einer inklusiven Perspektive verlangt ein Leistungsangebot für behinderte Kinder und Jugendliche, das sich primär an der Lebenslage ,Kindheit und Jugend" orientiert und erst sekundär nach der Behinderung oder anderen Benachteiligungen und Belastungen in dieser Lebenslage differenziert. Dies ist nach Auffassung der Bundesregierung der Maßstab, an dem die Leistungen für junge Menschen mit Behinderung zu messen sind."1

Fest steht, dass diese Forderung mitnichten neu ist ${ }^{2}$. Bezweifelt werden muss, ob die bis dato vorliegenden Dokumente der maßgeblichen politischen Akteure - darunter der zitierte 13. Kinder- und Jugendbericht, der Beschluss der 86. Arbeits- und Sozialministerkonferenz (ASMK) 200933, der Zwischenbericht der von der ASMK und Jugend- und Familienministerkonferenz (JFMK) eingesetzten Arbeitsgruppe „Inklusion von jungen Menschen mit Behinderung“ unter Beteiligung von Bund, Ländern, kommunalen Spitzenverbänden, der Bundesarbeitsgemeinschaft der Landesjugendämter und der Bundesarbeitsgemeinschaft der überörtlichen Sozialhilfeträger aus dem Jahre $2011^{4}$ - tatsächlich eine „Perspektive“ ihrem ursprünglichen Wortsinn nach eröffnen, der dem lateinischen „perspicere“ entstammt, was so viel bedeutet wie „mit dem Blick durchdringen, deutlich sehen, wahrnehmen“5.

Den Durchblick mag der eine oder andere bei der Debatte um die Eingliederungshilfe für Kinder und Jugendliche mit Behinderungen wohl eher verlieren. Zahlreich sind die in diesem Kontext sich ergebenden Probleme ${ }^{6}$, divers die Lösungsansätze ${ }^{7}$.

1 Bericht über die Lebenssituation junger Menschen und die Leistungen der Kinder- und Jugendhilfe in Deutschland - 13. Kinder- und Jugendbericht, BT-Drucks. 16/12860, S. 12.

2 Florian Gerlach/Knut Hinrichs, Inklusion und die „Große Lösung“ für die Jugend- und Behindertenhilfe (Teil 1), ZKJ 2012, S. 86.

3 Beschlussprotokoll der 86. Konferenz der Ministerinnen und Minister, Senatorinnen und Senatoren für Arbeit und Soziales der Länder, 2009, S. 11 f., abrufbar unter: http://www.sms.sachsen.de/ download/Verwaltung/ergebnis-asmk2009.pdf (letzter Zugriff: 6.5.2012).

4 Siehe das Beschlussprotokoll der 88. Konferenz der Ministerinnen und Minister, Senatorinnen und Senatoren für Arbeit und Soziales der Länder, 2011, S. 34 ff., abrufbar unter: http://www.sms. sachsen.de/download/Verwaltung/Protokoll_extern.pdf (letzter Zugriff: 5.6.2012).

5 Duden, Das Herkunftswörterbuch - Etymologie der deutschen Sprache, 4. Aufl. Mannheim 2007, S. 600.

6 Siehe etwa: Minou Banafsche, Kinder und Jugendliche mit Behinderung zwischen SGB VIII und SGB XII - im Lichte der UN-Behindertenrechtskonvention, ZKJ 2011, S. 116 ff.; Gerlach/Hinrichs (Fußn. 2), ZKJ 2012, S. 86 ff.; Zwischenbericht 2011 (Fußn. 4), S. 36 ff.

7 Eine Bewertung vornehmend: Stefan Greß/Stephan Rixen/Jürgen Wasem, Eingliederungshilfe für seelisch behinderte Kinder und Jugendliche: Abgrenzungsprobleme und Reformszenarien, VSSR 2009, S. 43, 51 ff.; ferner: Zwischenbericht 2011 (Fußn. 4), S. 24 ff., 28 ff. 
Ziel des vorliegenden Beitrags soll es sein, das „,duale System“ der Eingliederungshilfe für Kinder und Jugendliche mit Behinderungen auf seine Vereinbarkeit mit dem Gleichheitssatz des Art. 3 Grundgesetz (GG) zu überprüfen.

\section{Die Vereinbarkeit des ,,dualen Systems “ mit Art. 3 GG}

\section{Prüfungsmaßstab}

Denkbar wäre als Prüfungsmaßstab neben Art. 3 GG auch das Verbot der Diskriminierung aufgrund von Behinderung aus Art. 5 Abs. 2 UN-BRK, welches den allgemeinen Grundsatz der Nichtdiskriminierung aus Art. 3 Buchstabe a UN-BRK konkretisiert. Danach verbieten die Vertragsstaaten ,jede Diskriminierung aufgrund von Behinderung [...].“ Darunter fällt gem. Art. 2 Unter-Abs. 3 UN-BRK, ,jede Unterscheidung, Ausschließung oder Beschränkung aufgrund von Behinderung, die zum Ziel oder zur Folge hat, dass das auf die Gleichberechtigung mit anderen gegründete Anerkennen, Genießen oder Ausüben aller Menschenrechte und Grundfreiheiten im politischen, wirtschaftlichen, sozialen, kulturellen, bürgerlichen oder jedem anderen Bereich beeinträchtigt oder vereitelt wird. Sie umfasst jede Form der Diskriminierung, einschließlich der Versagung angemessener Vorkehrungen." 8 Letztere werden ihrerseits in Art. 2 Unter-Abs. 4 UNBRK definiert. 9

\section{a) Persönlicher Anwendungsbereich des Art. 5 Abs. 2 UN-BRK}

Eine Definition von „Behinderung“ enthält die UN-BRK nicht, insbesondere nicht in dem eigens mit „Begriffsbestimmungen“ („,definitions“) überschriebenen Art. 2 UNBRK. ${ }^{10}$ Allerdings eröffnet Art. 1 UN-BRK, der eigentlich den „Zweck“ (,purpose“) der Konvention zu regeln vorgibt, in Unter-Abs. 2 ihren persönlichen Anwendungsbereich. Danach zählen zu den Menschen mit Behinderungen solche, „die langfristige körperliche, seelische, geistige oder Sinnesbeeinträchtigungen haben, welche sie in Wechselwirkung mit verschiedenen Barrieren an der vollen, wirksamen und gleichberechtigten Teilhabe an der Gesellschaft hindern können." Einen Hinweis, warum von

8 Zum Grundsatz der Nichtdiskriminierung nach der UN-BRK: Valentin Aichele/Nina Althoff, NichtDiskriminierung und angemessene Vorkehrungen in der UN-Behindertenrechtskonvention, in: Antje Welke (Hrsg.), UN-Behindertenrechtskonvention mit rechtlichen Erläuterungen, Berlin 2012, S. 104, Rdnr. 5 ff.

$9 \mathrm{Zu}$ den angemessenen Vorkehrungen nach Art. 2 Unter-Abs. 4 UN-BRK: Aichele/Althoff (Fußn. 8), S. 104, Rdnr. $41 \mathrm{ff}$.

10 Theresia Degener, Welche legislativen Herausforderungen bestehen in Bezug auf die nationale Implementierung der UN-Behindertenrechtskonvention in Bund und Ländern?, br 2009, S. 34. 
einer Definition von Behinderung abgesehen wurde, gibt Buchstabe e der Präambel, dem die Erkenntnis zugrunde liegt, dass sich das Verständnis von Behinderung ständig weiterentwickelt. ${ }^{11}$ Angesichts dessen wollte man den Schutzbereich der UN-BRK nicht durch eine statische Begriffsbestimmung verengen. ${ }^{12}$

Da Art. 1 Unter-Abs. 2 UN-BRK weiter reicht als die Definition des § 2 Abs. 1 Satz 1 SGB IX ${ }^{13}$, auf den wiederum die im Falle von Kindern und Jugendlichen mit körperlichen und/oder geistigen Behinderungen maßgebliche Vorschrift des $§ 53$ Abs. 1 Satz 1 SGB XII verweist ${ }^{14}$ und auf den die den Anspruch auf Eingliederungshilfe für Kinder und Jugendliche mit seelischen Behinderungen gewährende Norm des § 35a Abs. 1 Satz 1 SGB VIII mittelbar rekurriert ${ }^{15}$, ist der persönliche Anwendungsbereich der UN-BRK vorliegend eröffnet.

\section{b) Sachlicher Anwendungsbereich des Art. 5 Abs. 2 UN-BRK}

Die UN-BRK unterscheidet nicht zwischen Behinderungsarten, sondern bezieht alle unter Art. 1 Unter-Abs. 2 fallenden Menschen gleichermaßen in ihren Schutzbereich ein. Zweck des Übereinkommens ist es gem. Art. 1 Unter-Abs. 1, „den vollen und

11 Gem. Art. 31 Abs. 2 Wiener Vertragsrechtskonvention (WVK) ist die Präambel eines völkerrechtlichen Vertrages für dessen Auslegung neben dem Vertragswortlaut, Zusammenhang sowie Sinn und Zweck des Vertrages ebenfalls von Bedeutung. Vgl. auch: Manfred Nowak, U.N. Covenant on Civil and Political Rights - CCPR Commentary, 2. Aufl. Kehl am Rhein 2005, Präambel Rdnr. 1.

12 Dazu: Theresia Degener, Die UN-Behindertenrechtskonvention als Inklusionsmotor, RdJB 2009, S. 200, 204; Theresia Degener, Die UN-Behindertenrechtskonvention - Grundlage für eine neue inklusive Menschenrechtstheorie, VN 2010, S. 57 f.; zu dem Streitstand während des Entstehungsprozesses der Konvention: Theresia Degener, Menschenrechtsschutz für behinderte Menschen - Vom Entstehen einer neuen Menschenrechtskonvention der Vereinten Nationen, VN 2006, S. 104, 106; Jochen von Bernstorff, Menschenrechte und Betroffenenrepräsentation: Entstehung und Inhalt eines UN-Antidiskriminierungsübereinkommens über die Rechte von behinderten Menschen, ZaöRV 67 (2007), S. 1041, 1047.

13 Siehe die Gegenüberstellung von Art. 1 Unter-Abs. 2 UN-BRK und $\S 2$ Abs. 1 SGB IX bei: Minou Banafsche, Die UN-Behindertenrechtskonvention und das deutsche Sozialrecht - eine Vereinbarkeitsanalyse anhand ausgewählter Beispiele (Teil I), SGb 2012, S. 373, $374 \mathrm{ff}$.

14 Einen eigenständigen, von $\S 2$ Abs. 1 Satz 1 SGB IX abweichenden Behinderungsbegriff implementiert $\S 53$ Abs. 1 Satz 1 SGB XII daher nicht. Siehe: Felix Welti, in: Klaus Lachwitz/Walter Schellhorn/ders. (Hrsg.), HK-SGB IX, 3. Aufl. Köln 2010, § 2 Rdnr. 10; Eckart Stevens-Bartol, in: Werner Feldes/Wolfhard Kohte/ders. (Hrsg.), SGB IX, Frankfurt am Main 2009, § 2 Rdnr. 5; Bernhard Knittel, SGB IX, 6. Aufl. Köln 2012, § 2 Rdnr. 9; Hartmut Haines, in: Dirk Dau/Franz Josef Düwell/Jacob Joussen (Hrsg.), LPK-SGB IX, 3. Aufl. Baden-Baden 2011, § 2 Rdnr. 15; von einem einheitlichen Behinderungsbegriff des $\S 2$ Abs. 1 Satz 1 SGB IX gehen ebenfalls aus: Volker Wahrendorf, in: Christian Grube/ders. (Hrsg.), SGB XII, 4. Aufl. München 2012, § 53 Rdnr. 13; Renate Bieritz-Harder, in: dies./Wolfgang Conradis/Stephan Thie (Hrsg.), LPK-SGB XII, 9. Aufl. Baden-Baden 2012, § 53 Rdnr. 3 ff., 11.

15 Vgl. Thomas Meysen, in: Johannes Münder/ders./Thomas Trenczek (Hrsg.), Frankfurter Kommentar zum SGB VIII, 6. Aufl. Baden-Baden 2009, § 35a Rdnr. 6, 16; Reinhard Wiesner, in: ders. (Hrsg.), SGB VIII, 4. Aufl. München 2011, § 35a Rdnr. 3, 5 f. 
gleichberechtigten Genuss aller Menschenrechte und Grundfreiheiten durch alle Menschen mit Behinderungen zu fördern, zu schützen und zu gewährleisten und die Achtung der ihnen innewohnenden Würde zu fördern." Verboten ist demgemäß nach Art. 5 Abs. 2 UN-BRK, ,jede Diskriminierung aufgrund von Behinderung“. Die Passus „,durch alle Menschen mit Behinderungen“ und ,jede Diskriminierung aufgrund von Behinderung" erlauben indes nicht nur eine Blickrichtung, die die Gruppe der Menschen mit Behinderungen der Gruppe der Menschen ohne Behinderungen gegenüberstellt, sondern zudem eine solche, die ungerechtfertigte Unterscheidungen innerhalb der Gruppe der Menschen mit Behinderungen verbietet.

Dies gilt umso mehr, als „,die Achtung vor der Unterschiedlichkeit von Menschen mit Behinderungen“ (,respect for difference“) im Kontext mit der „,menschlichen Vielfalt" (,human diversity“) die Individualität von Menschen mit Behinderungen zum allgemeinen Grundsatz der Konvention erhebt (vgl. Art. 3 Buchstabe d UN-BRK, Buchstabe i der Präambel). Dem wird auch durch die teilweise erfolgte Bezugnahme auf bestimmte Beeinträchtigungen in einzelnen Vorschriften Rechnung getragen, zum Beispiel in Art. 9 Abs. 2 UN-BRK, der in öffentlichen Gebäuden explizit Beschilderungen in Brailleschrift (Buchstabe d) oder das Zurverfügungstellen von Gebärdendolmetschern verlangt (Buchstabe e), um Zugangsbarrieren abzubauen oder nicht zur Entstehung gelangen zu lassen, oder in Art. 24 Abs. 3 Satz 2 Buchstabe c UN-BRK, der zu geeigneten Maßnahmen gegenüber blinden, gehörlosen oder taubblinden Menschen verpflichtet, um diesen eine bestmögliche schulische und soziale Entwicklung zu ermöglichen. Gemäß dem Buchstaben h der Präambel liegt dem Übereinkommen die Erkenntnis zugrunde, „dass jede Diskriminierung aufgrund von Behinderung eine Verletzung der Würde und des Wertes darstellt, die jedem Menschen innewohnen [...].“ Der Schutz vor Diskriminierungen von Menschen mit Behinderungen vermittelt somit einen über einen bloß gruppenbezogenen Schutz hinausgehenden Individualschutz.

Wenn demnach der sich als Synthese der Maßgaben des Art. 1 Unter-Abs. 1 und des Art. 5 Abs. 2 UN-BRK darstellende Art. 4 Abs. 1 Satz 1 UN-BRK den Vertragsstaaten auferlegt, „die volle Verwirklichung aller Menschenrechte und Grundfreiheiten für alle Menschen mit Behinderungen ohne Diskriminierung aufgrund von Behinderung zu gewährleisten und zu fördern“ und zu diesem Zweck ,alle geeigneten Gesetzgebungs-, Verwaltungs- und sonstigen Maßnahmen zur Umsetzung der in diesem Übereinkommen anerkannten Rechte zu treffen“ (Art. 4 Abs. 1 Satz 2 Buchstabe a UN-BRK) ${ }^{16}$, ebenso

16 Daraus i. V. m. Art. 4 Abs. 5 UN-BRK folgt, dass Verpflichtungsadressaten Legislative, Exekutive und Judikative auf den Ebenen von Bund, Ländern und Kommunen gleichermaßen sind. Freilich ergibt sich dies letztlich schon aus Art. 20 Abs. 3 GG, wonach die Gesetzgebung an die verfassungsmäßige Ordnung gebunden ist und vollziehende Gewalt und Rechtsprechung an Gesetz und Recht gebunden sind. Vgl. zum Ganzen auch: Valentin Aichele, Die UN-Behindertenrechtskonvention und ihr Fakultativprotokoll - Ein Beitrag zur Ratifikationsdebatte, Policy Paper No. 9, Berlin 2008, S. 8; Valentin Aichele, Behinderung und Menschenrechte: Die UN-Konvention über die Rechte von Menschen mit Behinderungen, APuZ 23/2010, S. 13, 17; zu der Rolle der Kommunen 
„zur Änderung oder Aufhebung bestehender Gesetze, Verordnungen, Gepflogenheiten und Praktiken [...], die eine Diskriminierung von Menschen mit Behinderungen darstellen“" (Art. 4 Abs. 1 Satz 2 Buchstabe b UN-BRK), bedeutet dies die Verpflichtung, ihre Rechtsordnungen unbeschadet relationaler Erwägungen unter anderem auf solche Regelungen zu überprüfen, die an das Merkmal der Behinderung spezifische Rechtsfolgen knüpfen, um sodann ihre unter Umständen diskriminierende Wirkung zu eruieren.

\section{c) Das Verhältnis der UN-BRK zum Grundgesetz}

Vor dem Hintergrund der vorstehenden Ausführungen tangiert die unterschiedliche gesetzliche Behandlung von Kindern und Jugendlichen mit Behinderungen je nach Behinderungsart den Schutzbereich des Art. 5 Abs. 2 UN-BRK.

Gemäß dem Grundsatz der Völkerrechtsfreundlichkeit, dem die deutsche Rechtsordnung im Gesamten und mit ihr das Grundgesetz folgt ${ }^{17}$, gilt es, das einfache wie das Verfassungsrecht im Sinne der für Deutschland verbindlichen völkerrechtlichen Normen - völkerrechtskonform - zu interpretieren. ${ }^{18}$ Die Grenzen der Auslegung bilden dabei - entsprechend den Grundsätzen verfassungskonformer Auslegung 19 - Wortlaut

als Träger öffentlicher Gewalt und Teil der Exekutive: BVerfGE 73, 118, 191; 83, 37, 53 f.; vgl. auch: Bodo Pieroth, in: Hans Jarass/ders., GG, 12. Aufl. München 2012, Art. 28 Rdnr. 10.

17 Die Völkerrechtsfreundlichkeit des Grundgesetzes folgt namentlich aus Art. 1 Abs. 2, Art. 24 bis 26 und 100 Abs. 2 GG. Vgl.: Karl-Peter Sommermann, Völkerrechtlich garantierte Menschenrechte als Maßstab der Verfassungskonkretisierung - Die Menschenrechtsfreundlichkeit des Grundgesetzes, AöR 114 (1989), S. 391, 417; Karl-Peter Sommermann, in: Hermann von Mangoldt/Friedrich Klein/Christian Starck (Hrsg.), Grundgesetz, Bd. 2, 6. Aufl. München 2010, Art. 20 Rdnr. 254; vgl. auch: BVerfGE 111, 307, $317 \mathrm{f}$.

18 Sommermann, in: von Mangoldt/Klein/Starck (Fußn. 17), Art. 20 Rdnr. 254; Rudolf Geiger, Grundgesetz und Völkerrecht, 5. Aufl. München 2010, § 38 II.; Albert Bleckmann, Die Völkerrechtsfreundlichkeit der deutschen Rechtsordnung, DÖV 1979, S. 309, 312 f.; Albert Bleckmann, Der Grundsatz der Völkerrechtsfreundlichkeit der deutschen Rechtsordnung, DÖV 1996, S. 137, 140 f.; Thomas Giegerich, Wirkung und Rang der EMRK in den Rechtsordnungen der Mitgliedstaaten, in: Rainer Grote/Thilo Marauhn (Hrsg.), EMRK/GG - Konkordanzkommentar zum europäischen und deutschen Grundrechtsschutz, Tübingen 2006, Kap. 2 Rdnr. 19 ff.; Robert Uerpmann, Die Europäische Menschenrechtskonvention und die deutsche Rechtsprechung - Ein Beitrag zum Thema Völkerrecht und Landesrecht, Berlin 1993, S. 109 ff.; ferner: BVerfGE 74, 358, 370; 111, 307, 317 f.; 128, 326, 366 ff.; spezifisch zur UN-BRK: BVerfGE 128, 282, 306.

$19 \mathrm{Zu}$ den Grenzen verfassungskonformer Auslegung: BVerfGE 8, 28, 34; 8, 38, 41; 18, 97, 111;54, 277, 299 f.; 71, 81, 105; 90, 263, 275; 95, 64, 93; 110, 226, 267; 118, 212, 234; 119, 247, 274; ferner: Sommermann, in: von Mangoldt/Klein/Starck (Fußn. 17), Art. 20 Rdnr. 260; Christian Starck, in: Hermann von Mangoldt/Friedrich Klein/ders. (Hrsg.), Grundgesetz, Bd. 1, 6. Aufl. München 2010, Art. 1 Rdnr. 328; Christian Hillgruber, Richterliche Rechtsfortbildung als Verfassungsproblem, JZ 1996, S. 118, 119; Andreas Voßkuhle, Theorie und Praxis der verfassungskonformen Auslegung von Gesetzen durch Fachgerichte - Kritische Bestandsaufnahme und Versuch einer Neubestimmung, AöR 125 (2000), S. 177, 193 f. 
und erkennbarer Zweck der infrage stehenden Normen. ${ }^{20} \mathrm{Zu}$ berücksichtigen ist allerdings, dass völkerrechtliche Verträge gem. Art. 59 Abs. 2 Satz 1 GG jeweils durch Bundesgesetz in deutsches Recht überführt werden ${ }^{21}$ und selbst den Rang einfachen Bundesrechts einnehmen ${ }^{22}$. Aus diesem Grund sind völkerrechtliche Gewährleistungen kein unmittelbarer verfassungsrechtlicher Prüfungsmaßstab ${ }^{23}$, „beeinflussen jedoch die Auslegung der Grundrechte und rechtsstaatlichen Grundsätze des Grundgesetzes“ und „dienen auf der Ebene des Verfassungsrechts als Auslegungshilfen für die Bestimmung von Inhalt und Reichweite von Grundrechten und rechtsstaatlichen Grundsätzen des Grundgesetzes, sofern dies nicht zu einer selbst nicht gewollten ${ }^{24}[\ldots]$ Einschränkung oder Minderung des Grundrechtsschutzes nach dem Grundgesetz führt [...].“25 Das Grundgesetz ist, wie es das Bundesverfassungsgericht (BVerfG) formuliert, „nicht die weitesten Schritte der Öffnung für völkerrechtliche Bindungen gegangen“, zum einen mit Blick auf die Erforderlichkeit eines Zustimmungsgesetzes nach Art. 59 Abs. 2 Satz $1 \mathrm{GG}$, um völkerrechtlichen Verträgen zur innerstaatlichen Geltung zu verhelfen, zum anderen, weil es das Völkervertragsrecht „nicht mit dem Rang des Verfassungsrechts ausgestattet“ und somit nicht ,auf die in dem letzten Wort der deutschen Verfassung liegende Souveränität" verzichtet hat. ${ }^{26}$

20 Siehe etwa: Sommermann, AöR 114 (Fußn. 17), S. 391, 418; Sommermann, in: von Mangoldt/Klein/Starck (Fußn. 17), Art. 20 Rdnr. 254; vgl. auch: Bleckmann, DÖV 1979 (Fußn. 18), S. 309, 312.

21 Im Falle der UN-BRK durch das „Gesetz zu dem Übereinkommen der Vereinten Nationen vom 13. Dezember 2006 über die Rechte von Menschen mit Behinderungen sowie zu dem Fakultativprotokoll vom 13. Dezember 2006 zum Übereinkommen der Vereinten Nationen über die Rechte von Menschen mit Behinderungen" vom 21.12.2008, BGBl. II 2008, S. 1419.

22 BVerfGE 74, 358, 370; 111, 307, 317; exemplarisch: Bernhard Kempen, in: von Mangoldt/Klein/Starck (Fußn. 17), Art. 59 Rdnr. 92. Nach der sogenannten „Vollzugslehre“ bleibt völkerrechtlichen Verträgen aber auch nach der Überführung in deutsches Recht ihr völkerrechtlicher Charakter erhalten. Dazu und zu der Abgrenzung von der sogenannten „Transformationslehre“, nach der in deutsches Recht überführte völkerrechtliche Verträge ihren völkerrechtlichen Charakter verlieren, m. w. N.: Jochen von Bernstorff, Anmerkungen zur innerstaatlichen Anwendbarkeit ratifizierter Menschenrechtsverträge: Welche Rechtswirkungen erzeugt das Menschenrecht auf inklusive Schulbildung aus der UN-Behindertenrechtskonvention im deutschen Sozial- und Bildungsrecht?, RdJB 2011, S. 203, 204 ff.

23 BVerfGE 10, 271, 274; 34, 384, 395; 41, 126, 149; 64, 135, 157; 74, 102, 128; 111, 307, 317.

24 Siehe Art. 4 Abs. 4 Satz 2 UN-BRK, wonach ,die in einem Vertragsstaat durch Gesetze, Übereinkommen, Verordnungen oder durch Gewohnheitsrecht anerkannten oder bestehenden Menschenrechte und Grundfreiheiten [...] nicht unter dem Vorwand beschränkt oder außer Kraft gesetzt werden [dürfen], dass dieses Übereinkommen derartige Rechte oder Freiheiten nicht oder nur in einem geringeren Ausmaß anerkenne.“

25 BVerfGE 111, 307, 317; in der Sache ebenso: BVerfGE 74, 358, 370.

26 BVerfGE 111, 307, $318 \mathrm{f}$. 


\section{d) Ergebnis}

Aus den genannten Gründen, ihr Verhältnis zum Grundgesetz betreffend, soll vorliegend nicht die UN-BRK, sondern eben jenes als Prüfungsmaßstab herangezogen werden. Hierbei wird indes zu berücksichtigen sein, dass die verfassungsrechtlichen Gewährleistungen - „im Rahmen des demokratischen und rechtsstaatlichen Systems des Grundgesetzes“"27 - im Lichte der UN-BRK zu interpretieren sein werden. ${ }^{28}$

\section{Vereinbarkeit der dualen Eingliederungshilfe mit Art. 3 GG}

Gleichheitsrechtlicher Anknüpfungspunkt für die unterschiedliche gesetzliche Behandlung von Kindern und Jugendlichen mit Behinderungen je nach Behinderungsart könnte sowohl der allgemeine Gleichheitssatz des Art. 3 Abs. 1 GG als auch der besondere Gleichheitssatz des Art. 3 Abs. 3 Satz 2 GG sein, wonach niemand wegen seiner Behinderung benachteiligt werden darf. ${ }^{29}$ Der maßgebliche Unterschied liegt in den höheren Anforderungen des Art. 3 Abs. 3 Satz 2 GG an eine verfassungsrechtliche Rechtfertigung der Ungleichbehandlung. ${ }^{30}$

27 BVerfGE 111, 307, 318

28 Diese Form der gegenseitigen Beeinflussung von Grundgesetz und Völkervertragsrecht ähnelt strukturell der sogenannten „Wechselwirkungstheorie“ von Freiheitsrechten aus Art 5 Abs. 1 GG und den allgemeinen Gesetzen nach Art. 5 Abs. 2 GG als Grundrechtsschranken. So ist „die gegenseitige Beziehung zwischen Grundrecht und ,allgemeinem Gesetz' [...] nicht als einseitige Beschränkung der Geltungskraft des Grundrechts durch die ,allgemeinen Gesetze' aufzufassen; es findet vielmehr eine Wechselwirkung in dem Sinne statt, daß die ,allgemeinen Gesetze` zwar dem Wortlaut nach dem Grundrecht Schranken setzen, ihrerseits aber aus der Erkenntnis der wertsetzenden Bedeutung dieses Grundrechts im freiheitlichen demokratischen Staat ausgelegt und so in ihrer das Grundrecht begrenzenden Wirkung selbst wieder eingeschränkt werden müssen“, BVerfGE 7, 198, 208 f.; ferner: BVerfGE 59, 231, 265; 60, 234, 240; 61, 1, 10 f.; 66, 116, 150; 71, 206, 214; von dem Erfordernis eines „Abwägungsprozess[es] am Maßstab des Verhältnismäßigkeitsgrundsatzes“ im Rahmen der Wechselwirkungslehre spricht: Klaus Stern, Das Staatsrecht der Bundesrepublik Deutschland, Bd. IV/1, München 2006, § 108, IV 7 (S. 1476); ferner: Hans Jarass, in: ders./Pieroth (Fußn. 16), Art. 5 Rdnr. 57; Herbert Bethge, in: Michael Sachs (Hrsg.), Grundgesetz, 6. Aufl. München 2011, Art. 5 Rdnr. 146.

29 Zur Verantwortung des Staates in diesem Kontext: Hans Friedrich Zacher, Der soziale Rechtsstaat in der Verantwortung für Menschen mit Behinderungen, in: Ulrich Becker/Franz Ruland (Hrsg.), Abhandlungen zum Sozialrecht II, Heidelberg 2008, S. $175 \mathrm{ff}$.

30 Dazu unten II. 2. c). 


\section{a) Gleichheitsrechtlicher Prüfungsmaßstab}

Art. 3 Abs. 3 Satz 2 GG verbietet eine Benachteiligung ,wegen“ einer Behinderung, mithin eine Benachteiligung, die an das Merkmal der Behinderung anknüpft. ${ }^{31}$ Das Benachteiligungsverbot des Art. 3 Abs. 3 Satz 2 GG ist somit einschlägig, wenn eine Regelung eine bestimmte Rechtsfolge pauschal an den Tatbestand der „Behinderung“32 oder an eine bestimmte Art von Behinderung knüpft, wenn die Vergleichsgruppe Menschen ohne Behinderungen sind 33 .

In der vorliegenden Konstellation der dualen Eingliederungshilfe für Kinder und Jugendliche mit Behinderungen werden die Ansprüche auf Eingliederungshilfe jeweils an eine Behinderungsart geknüpft, allerdings ist die maßgebliche Vergleichsgruppe nicht die der Menschen ohne Behinderungen, sondern die der Menschen mit einer anderen Behinderungsart. Überwiegend wird in diesem Zusammenhang eine Benachteiligung wegen einer Behinderung mit dem Argument abgelehnt, es handele sich nur um eine Differenzierung innerhalb der Gruppe von Menschen mit Behinderungen ${ }^{34}$, und auf Art. 3 Abs. 1 GG zurückgegriffen ${ }^{35}$. Dem liegt offenbar der gedankliche Automatismus zugrunde, es gehe dem besonderen Gleichheitssatz darum, die als Einheit betrachtete Gruppe der „Behinderten“ gegenüber den „Nichtbehinderten“ zu stärken.

Zwar mögen diese Erwägungen bei der Schaffung des Art. 3 Abs. 3 Satz 2 GG im Vordergrund gestanden haben, ein zwingender Gegenschluss, dass Benachteiligungen innerhalb der Gruppe von Menschen mit Behinderungen per se aus dem Schutzbereich des Art. 3 Abs. 3 Satz 2 GG ausgeschlossen sind, lässt sich dem indes nicht entnehmen. ${ }^{36}$ Beschlussempfehlung und Bericht des Rechtsausschusses zu dem „Entwurf eines Gesetzes zur Änderung des Grundgesetzes der Fraktionen der CDU/CSU, SPD und F.D.P.“"37 enthalten keine Ausführungen zu einer Beschränkung des Art. 3 Abs. 3 Satz 2 GG auf das Verhältnis zwischen Menschen mit Behinderungen und Menschen ohne Behinderungen. Im Vordergrund stand das Ziel, gesellschaftliche und rechtliche Aus-

31 Guy Beaucamp, Das Behindertengrundrecht (Art. 3 Abs. 3 Satz 2 GG) im System der Grundrechtsdogmatik, DVBl. 2002, S. 997, 998; Felix Welti, Behinderung und Rehabilitation im sozialen Rechtsstaat, Tübingen 2005, S. 446 f.; Volker Neumann, in: Olaf Deinert/ders. (Hrsg.), Rehabilitation und Teilhabe behinderter Menschen - Handbuch SGB IX, 2. Aufl. Baden-Baden 2009, § 2 Rdnr. 24.

32 Beispielhaft: Welti (Fußn. 31), S. 467.

33 Lerke Osterloh, in: Sachs (Fußn. 28), Art. 3 Rdnr. 311.

34 So etwa: Rupert Scholz, in: Theodor Maunz/Günter Dürig (Begr.), Grundgesetz, Bd. I, 64. Ergänzungslieferung, Stand: Januar 2012, München 2012, Art. 3 Rdnr. 174 (Fußn. 1); Osterloh, in: Sachs (Fußn. 28), Art. 3 Rdnr. 311.

35 Siehe: Neumann, in: Deinert/ders. (Fußn. 31), § 2 Rdnr. 20, der Art. 3 Abs. 1 GG für den „flexiblere[n] Prüfungsmaßstab" erachtet.

36 Dazu, dass Gegenschlüsse nicht per se zwingend sind: Ulrich Klug, Juristische Logik, 4. Aufl. Berlin 1982, S. 137 ff.; Karl Larenz, Methodenlehre der Rechtswissenschaft, 5. Aufl. Berlin 1983, S. 374 .

37 BT-Drucks. 12/6633. 
grenzungen von Menschen mit Behinderungen zu verhindern. ${ }^{38}$ Eine darüber hinausgehende Einigkeit zwischen den verschiedenen Fraktionen im Detail bestand hingegen nicht. ${ }^{39}$ Dass dabei von der „Gruppe der Behinderten“ die Rede war, belegt nicht, dass diese per se als geschlossene Gruppe betrachtet wurde, sondern nur, dass alle Menschen mit Behinderungen an dem besonderen Diskriminierungsschutz partizipieren sollten. Als Ausdruck der Wertordnung des Grundgesetzes verstärke aus Sicht der Fraktion der SPD „das Benachteiligungs[verbot] den sozialstaatlichen Auftrag, die Voraussetzungen grundrechtlicher Freiheit und eines menschenwürdigen Daseins zu sichern und auf eine gleichberechtigte Teilhabe behinderter Menschen in der Gesellschaft hinzuwirken."40 Die Menschenwürde ist aber nun, unbeschadet der Schwierigkeit einer Schutzbereichsbestimmung ${ }^{41}$, stets subjektbezogen ${ }^{42}$ : So wird sie zum Teil als naturgegebener Eigenwert eines jeden Menschen begriffen, der ihm qua Menschsein zukommt ${ }^{43}$, zum Teil als erst zu erreichendes Ergebnis von Selbstdarstellung in der Kommunikation mit anderen $^{44}$. Das BVerfG erachtet es ,ausgehend von der Vorstellung des Grundgesetzgebers, dass es zum Wesen des Menschen gehört, in Freiheit sich selbst zu bestimmen und sich frei zu entfalten, und dass der Einzelne verlangen kann, in der Gemeinschaft grundsätzlich als gleichberechtigtes Glied mit Eigenwert anerkannt zu werden", für durch die Verpflichtung zur Achtung und zum Schutz der Menschenwürde generell ausgeschlossen, „den Menschen zum bloßen Objekt des Staates zu machen [...]. Schlechthin verboten ist damit jede Behandlung des Menschen durch die öffentliche Gewalt, die dessen Subjektqualität, seinen Status als Rechtssubjekt, grundsätzlich in Frage stellt [...], indem sie die Achtung des Wertes vermissen lässt, der jedem Menschen um seiner selbst willen, kraft seines Personseins, zukommt [...].“45

38 BT-Drucks. 12/8165, S. 28.

39 Vgl.: BT-Drucks. 12/8165, S. 29.

40 BT-Drucks. 12/8165, S. 29.

41 Zusammenfassend: Bodo Pieroth/Bernhard Schlink, Grundrechte - Staatsrecht II, 28. Aufl. Heidelberg 2012, Rdnr. 368 ff.

42 Von dem „Prinzip der Personhaftigkeit des Menschen“ spricht: Hasso Hofmann, Die versprochene Menschenwürde, AöR 1993, S. 353, 358, 364.

43 In der Sache bejahend, allerdings den Gemeinschaftsbezug des Menschen hervorhebend, um nicht Gefahr zu laufen, den Satz von der Würde der menschlichen Persönlichkeit „zur magna charta des Individualismus zu machen“: Günter Dürig, Die Menschenauffassung des Grundgesetzes, JR 1952, S. 259, 261; den Eigenwert des Menschen herausstellend: Hans Carl Nipperdey, Die Würde des Menschen, in: Franz Neumann/ders./Ulrich Scheuner (Hrsg.), Die Grundrechte - Handbuch der Theorie und Praxis der Grundrechte, Bd. II, Berlin 1954, S. 1 ff., der Art. 1 Abs. 1 GG als ,naturrechtliches Elementarprinzip“ begreift; Starck, in: von Mangoldt/Klein/ders. (Fußn. 19), Art. 1 Rdnr. 11; kritisch gegenüber einer überpositiven Deutung der Menschenwürdegarantie: Matthias Herdegen, in: Maunz/Dürig (Fußn. 34), Art. 1 Rdnr. 19 f.

44 Niklas Luhmann, Grundrechte als Institution - Ein Beitrag zur politischen Soziologie, 2. Aufl. Berlin 1974, S. $68 \mathrm{f}$.

45 BVerfGE 115, 118, 153; vgl. ferner: BVerfGE 45, 187, 227 f.; 87, 209, 228; 96, 375, 399 f.; 109, 279, 312 f.; vgl. bereits: BVerfGE 30, 1, 26, wo das BVerfG seine „Objektformel“ erweitert hat, die 
Dafür, dass Art. 3 Abs. 3 Satz 2 GG weniger als kollektiv denn als individuell ausgerichtetes Recht zu begreifen ist, spricht auch der Wortlaut, ausweislich dessen niemand wegen ,seiner“ Behinderung benachteiligt werden darf. ${ }^{46}$

Gestützt wird dieses Ergebnis schließlich durch die zu Art. 5 Abs. 2 UN-BRK gemachten Ausführungen ${ }^{47}$, die in die Auslegung des Grundrechts aus Art. 3 Abs. 3 Satz 2 GG im Wege völkerrechtskonformer Interpretation 48 einfließen.

Als gleichheitsrechtlicher Prüfungsmaßstab wird hier folglich Art. 3 Abs. 3 Satz 2 GG herangezogen.

\section{b) Rechtlich relevante Ungleichbehandlung}

Da sich die Zuständigkeit und die Voraussetzungen für die Leistungen zur Teilhabe gem. § 7 Satz 2 SGB IX, § 53 Abs. 4 Satz 2 SGB XII nach den für den jeweiligen Rehabilitationsträger geltenden Leistungsgesetzen richten, wirkt sich die gesetzliche Aufteilung von Kindern und Jugendlichen mit Behinderungen auf zwei Sozialleistungssysteme rechtlich vor allem in den Bereichen des Leistungsrechts und der Zuständigkeit aus. 49

\section{aa) Zuständigkeitsdivergenzen}

Zuständig für die Eingliederungshilfe nach dem SGB VIII sind mangels Zuständigkeit der überörtlichen Träger gem. $\S 85$ Abs. 2 SGB VIII ${ }^{50}$ die örtlichen Träger der Jugendhilfe ( 885 Abs. 1 SGB VIII). Die Träger der öffentlichen Jugendhilfe werden gem. $\S 69$ Abs. 1 SGB VIII durch Landesrecht bestimmt.

In der Sozialhilfe sind gem. $\S 97$ Abs. 1 SGB XII ebenfalls die örtlichen Träger gem. § 3 Abs. 2 Satz 1 SGB XII sind dies vorbehaltlich anderslautender landesrechtlicher Bestimmungen die Kreise und kreisfreien Städte - sachlich zuständig, soweit nicht die überörtlichen Träger sachlich zuständig sind. Allerdings wird gem. § 97 Abs. 2 Satz 1 SGB XII die sachliche Zuständigkeit des überörtlichen Trägers durch Landesrecht bestimmt wie gem. § 3 Abs. 3 SGB XII auch, wer überörtlicher Träger der Sozialhilfe

zunächst allein auf das Verbot einer Objektivation des Menschen rekurrierte. Siehe etwa: BVerfGE 27, 1,6 .

46 Welti (Fußn. 31), S. 470; zur Heterogenität von Gruppen und Individualität von „Gruppenrechten“: Rainer Nickel, Gleichheit und Differenz in der vielfältigen Republik - Plädoyer für ein erweitertes Antidiskriminierungsrecht, Baden-Baden 1999, S. 63 f.

47 Siehe II.1.b).

48 Dazu oben II.1.c).

49 Siehe dazu bereits: Banafsche (Fußn. 6), ZKJ 2011, S. 116, 119 f., 121 f.; Minou Banafsche, Die UN-Behindertenrechtskonvention und das deutsche Sozialrecht - eine Vereinbarkeitsanalyse anhand ausgewählter Beispiele (Teil II), SGb 2012, S. 440, 443.

50 Ihnen obliegt im Rahmen der Eingliederungshilfe für seelisch behinderte Kinder und Jugendliche gem. $§ 85$ Abs. 2 Nr. 2 SGB VIII eine fördernde Funktion. Vgl. auch: Gila Schindler, in: Münder/Meysen/Trenczek (Fußn. 15), § 85 Rdnr. 3; Wiesner, in: ders. (Fußn. 15), § 85 Rdnr. 12 ff. 
ist. Dies hat im Rahmen der Eingliederungshilfe nach dem SGB XII innerhalb einiger Länder ${ }^{51}$ eine geteilte Zuständigkeit für ambulante und stationäre Leistungen hervorgebracht.

\section{bb) Leistungsrechtliche Divergenzen}

Während § 35a Abs. 1 Satz 1 SGB VIII für die Gewährung eines Anspruchs auf Eingliederungshilfe allein das Vorliegen einer seelischen Behinderung verlangt, erhalten Leistungen der Eingliederungshilfe auf Grundlage des $\S 53$ Abs. 1 Satz 1 SGB XII nur Personen, die durch eine Behinderung i. S. d. § 2 Abs. 1 Satz 1 SGB IX ,wesentlich in ihrer Fähigkeit, an der Gesellschaft teilzuhaben, eingeschränkt oder von einer solchen wesentlichen Behinderung bedroht sind [...], wenn und solange nach der Besonderheit des Einzelfalles, insbesondere nach Art oder Schwere der Behinderung, Aussicht besteht, dass die Aufgabe der Eingliederungshilfe erfüllt werden kann." Gem. § 19 Abs. 3 SGB XII werden zudem Leistungen der Eingliederungshilfe nur gewährt, „soweit den Leistungsberechtigten $[\ldots]$ und, wenn sie minderjährig und unverheiratet sind, auch ihren Eltern oder einem Elternteil die Aufbringung der Mittel aus dem Einkommen und Vermögen nach den Vorschriften des Elften Kapitels dieses Buches nicht zuzumuten ist."

Der sozialhilferechtliche Anspruch des $\S 53$ Abs. 1 Satz 1 i. V. m. § 19 Abs. 3 SGB XII setzt somit über das Vorliegen einer Behinderung hinaus die Bedürftigkeit des Betroffenen voraus, mithin das Unvermögen, seinen Lebensunterhalt aus eigenen Kräften $\mathrm{zu}$ bestreiten oder sich in der bestehenden Lebenslage selbst zu helfen ${ }^{52}$.

Des Weiteren bedarf es gem. $\S 53$ Abs. 1 Satz 1 SGB XII einer „wesentlichen“ Behinderung. Darüber, was unter einer wesentlichen körperlichen und wesentlichen geistigen Behinderung zu verstehen ist, gibt die auf Grundlage des $\S 60$ SGB XII erlassene Eingliederungshilfe-Verordnung (EinglH-VO) in den $\S \S 1$ und 2 Aufschluss. ${ }^{53}$

Schließlich wird Eingliederungshilfe nach $\S 53$ Abs. 1 Satz 1 Halbsatz 2 SGB XII im Sinne einer Erfolgsbezogenheit nur geleistet, „wenn und solange nach der Besonderheit

51 Namentlich sind dies Hessen, Niedersachsen, Nordrhein-Westfalen, Rheinland-Pfalz und Sachsen.

52 So: Wahrendorf, in: Grube/ders. (Fußn. 14), § 9 Rdnr. 28. Auf die vom Erfordernis der Bedürftigkeit als Leistungsvoraussetzung abstrakt zu betrachtende Einschränkung der Anrechnung von Einkommen und Vermögen bei Menschen mit Behinderungen nach Maßgabe der $\S \S 86,87$ Abs. 1 Sätze 2 und 3 SGB XII und des $\S 92$ SGB XII, gegebenenfalls auch des § 92a SGB XII, sei an dieser Stelle hingewiesen.

53 Das Vorliegen einer wesentlichen geistigen Behinderung kann insbesondere bei Teilleistungsstörungen, wie Legasthenie und Dyskalkulie, problematisch sein: Peter Scheider, in: Walter Schellhorn/Helmut Schellhorn/Karl-Heinz Hohm (Hrsg.), SGB XII, 18. Aufl. Köln 2010, § 2 EinglH-VO Rdnr. 4; Meysen, in: Münder/ders./Trenczek (Fußn. 15), § 35a Rdnr. 40 f.; Jörg Fegert, in: Wiesner (Fußn. 15), § 35a Rdnr. 71 ff. 
des Einzelfalls, insbesondere nach Art oder Schwere der Behinderung, Aussicht besteht, dass die Aufgabe der Eingliederungshilfe erfüllt werden kann." ${ }^{\text {"54 }}$

\section{cc) Ergebnis}

Leistungsrechtlich ist der Erhalt von Eingliederungshilfe nach dem SGB XII demnach von weiter gehenden Voraussetzungen abhängig als im Regelungskontext des SGB VIII, so dass im Ergebnis eine rechtlich relevante Ungleichbehandlung zu bejahen ist.

Die im Rahmen der Sozialhilfe in einigen Ländern geteilte Zuständigkeit führt dazu, dass Kinder und Jugendliche mit körperlichen und/oder geistigen Behinderungen sich dort bei einem Bedarf an sowohl ambulanten als auch stationären Leistungen an unterschiedliche Leistungsträger halten müssen und auch insoweit gegenüber Kindern und Jugendlichen mit seelischen Behinderungen benachteiligt sind. Da rechtfertigungsbedürftig nur eine rechtlich relevante Ungleichbehandlung von wesentlich Gleichem ist ${ }^{55}$ und eine „Gleichbehandlung nur innerhalb des Zuständigkeitsbereichs des jeweiligen Gesetzgebers" verlangt werden kann ${ }^{56}$, beschränkt sich im Hinblick auf die Zuständigkeit das Vorliegen einer rechtlich relevanten Ungleichbehandlung von Kindern und Jugendlichen mit körperlichen und/oder geistigen Behinderungen auf die Länder mit geteilter Zuständigkeit. 57

\section{c) Verfassungsrechtliche Rechtfertigung der Ungleichbehandlung}

Eine verfassungsrechtliche Rechtfertigung der Ungleichbehandlung im Rahmen des Art. 3 Abs. 3 Satz 2 GG kann nur bejaht werden, „wenn zwingende Gründe dafür vorliegen [...]. Die nachteiligen Auswirkungen müssen unerlässlich sein, um behinderungsbezogenen Besonderheiten Rechnung zu tragen [...]." ${ }^{58}$ Es bedarf demgemäß einer strengen Verhältnismäßigkeitsprüfung ${ }^{59}$, die dem gesetzgeberischen Gestaltungsspielraum enge Grenzen setzt ${ }^{60}$.

54 Siehe zu diesem Merkmal: Wahrendorf, in: Grubelders. (Fußn. 14), § 53 Rdnr. 31; Bieritz-Harder, in: LPK-SGB XII (Fußn. 14), § 53 Rdnr. 20; VG Meiningen RdLH 1999, S. 63, 64; LSG SachsenAnhalt FEVS 57 (2006), S. 553, $554 \mathrm{f}$.

55 Pieroth/Schlink (Fußn. 41), Rdnr. 463.

56 BVerfG NVwZ 1997, S. 1207; siehe bereits: BVerfGE 10, 354, 371; 12, 139, 143; 12, 319, 324; 21, 54,68 .

57 Zum weit verstandenen Begriff des Nachteils im Rahmen des Art. 3 Abs. 3 Satz 2 GG mit weiteren Nachweisen: Osterloh, in: Sachs (Fußn. 28), Art. 3 Rdnr. 312.

58 BVerfGE 99, 341, 357; Jarass, in: ders./Pieroth (Fußn. 16), Art. 3 Rdnr. 149.

59 Osterloh, in: Sachs (Fußn. 28), Art. 3 Rdnr. 314.

60 Vgl: Pieroth/Schlink (Fußn. 41), Rdnr. 481, 488. 


\section{aa) Zuständigkeitsdivergenzen}

$\S 97$ SGB XII normiert die sachliche Zuständigkeit für die gesamte Sozialhilfe und überantwortet es dem Landesgesetzgeber zu regeln, welche Bereiche der Sozialhilfe in die sachliche Zuständigkeit der überörtlichen Träger der Sozialhilfe fallen sollen (§97 Abs. 1 Halbsatz 2 und Abs. 2 Satz 1 SGB XII) und wer überörtlicher Träger ist (§ 3 Abs. 3 SGB XII). ${ }^{61}$ Als Teil des Verwaltungsorganisationsrechts dient $§ 97$ SGB XII der Verwirklichung des materiellen Rechts. ${ }^{62}$ Nach der nach Maßgabe des Art. 30 GG statuierten bundesstaatlichen Kompetenzverteilung als für das in Art. 20 Abs. 1 und Art. 28 Abs. 1 GG verankerte Bundesstaatsprinzip ,grundlegende Vorschrift“63 führen die Länder die Sozialhilfe als eigene Angelegenheit aus (Art. 30 i. V. m. Art. 83 Halbsatz 1 GG). Gem. Art. 84 Abs. 1 Satz 1 GG regeln sie im Rahmen der Landeseigenverwaltung unter anderem die Einrichtung der Behörden ${ }^{64}$, was neben deren Errichtung auch die Zuweisung von Aufgaben und Befugnissen, ,insbesondere die Festlegung der Zuständigkeit für die Gesetzesausführung"65, umfasst. ${ }^{66}$ Die Regelung der sachlichen Zuständigkeit durch die Länder ist damit unmittelbarer Ausdruck und Folge des föderalen Prinzips, mithin verfassungsimmanent, und erscheint unter gleichheitsrechtlichen Gesichtspunkten nicht problematisch.

Zwar „soll“ gem. § 97 Abs. 2 Satz 2 SGB XII berücksichtigt werden, dass unter anderem für Leistungen der Eingliederungshilfe ( 8 Nr. 4 SGB XII) „,so weit wie möglich [...] eine einheitliche sachliche Zuständigkeit gegeben ist." Entgegen der durch den Begriff ,soll“" entstehenden Vermutung erfordert eine uneinheitliche Regelung der sachlichen Zuständigkeit durch den Landesgesetzgeber keinen Ausnahmefall im Sinne einer atypischen Situation ${ }^{67} .68$ Vielmehr handelt es sich hierbei nur um eine Anregung ge-

61 Siehe bereits oben II.2.b)aa).

62 So: Wahrendorf, in: Grubelders. (Fußn. 14), § 97 Rdnr. 2.

63 BVerfGE 12, 205, 244; ferner: BVerfGE 36, 342, 365 f., wo Art. 30 GG als ,fundamentale Grundsatzbestimmung" aufgeführt wird; für das Schrifttum: Pieroth, in: Jarass/ders. (Fußn. 16), Art. 30 Rdnr. 1; Wilfried Erbguth, in: Sachs (Fußn. 28), Art. 30 Rdnr. 1; Wolfgang März, in: von Mangoldt/Klein/Starck (Fußn. 17), Art. 30 Rdnr. 1 f.

64 BVerfGE 10, 20, 48, wonach man unter einer Behörde ,im allgemeinen eine in den Organismus der Staatsgewalt eingeordnete, organisatorische Einheit von Personen und sächlichen Mitteln [versteht], die mit einer gewissen Selbständigkeit ausgestaltet dazu berufen ist, unter öffentlicher Autorität für die Erreichung der Zwecke des Staates oder von ihm geförderter Zwecke tätig zu sein.“ Dazu gehören auch die Kommunen, Kommunal- und Planungsverbände als Teil der Landesstaatsgewalt. Zur Rechtsnatur der Kommunen im Kontext mit Art. 83 GG: BVerfGE 39, 96, 109; aus dem Schrifttum: Ulrich Becker, Bayerisches Kommunalrecht, in: ders./Dirk Heckmann/Bernhard Kempen/Gerrit Manssen, Öffentliches Recht in Bayern, 5. Aufl. München 2011, 2. Teil Rdnr. 6 ff. (S. 74 f.).

65 BSGE 102, 149, 157, Rdnr. 39.

66 Vgl.: BVerfGE 105, 313, 331; BSGE 102, 149, 157, Rdnr. 39; implizit auch: BVerfGE 75, 108, $151 ; 126,77,99$.

67 Exemplarisch zu den „Soll“-Vorschriften: Hartmut Maurer, Allgemeines Verwaltungsrecht, 18. Aufl. München 2011, § 7 Rdnr. 11.

68 Bayerisches LSG, Urt. v. 31.7.2006 - L 11 SO 27/06 - Rdnr. 22 (zitiert nach juris). 
genüber dem jeweiligen Landesgesetzgeber ${ }^{69}$, der frei entscheiden kann, ob er dieser Folge leistet. ${ }^{70}$

\section{bb) Leistungsrechtliche Divergenzen}

Gegen eine verfassungsrechtliche Rechtfertigung der leistungsrechtlichen Divergenzen spricht zum einen die Normgenese des § 35a SGB VIII und sprechen zum anderen gesetzessystematische Erwägungen.

\section{(1) Die Normgenese des § 35a SGB VIII}

Die Normgenese des $\S 35$ a SGB VIII macht sichtbar, dass von Anfang an die Zuständigkeitsstreitigkeiten zwischen Jugend- und Sozialhilfe im Vordergrund standen, nicht hingegen sachliche Erwägungen für eine unterschiedliche leistungsrechtliche Behandlung von Kindern und Jugendlichen mit Behinderungen je nach Behinderungsart.

Ansprüche für Menschen mit seelischer Behinderung waren zunächst der Sozialhilfe vorbehalten; durch das Zweite Gesetz zur Änderung des Bundessozialhilfegesetzes vom 14. August 196971, gem. Art. $2 \S 9$ Abs. 1 in Kraft getreten am 1. Oktober 1969, wurde dem $\S 39$ Abs. 1 Satz 1 eine Nummer 6 angefügt, die Menschen erfasste, die ,seelisch wesentlich behindert" waren. Das Gesetz für Jugendwohlfahrt (JWG) vom 11. August $1961^{72}$ enthielt demgegenüber keine spezifischen Leistungen für Kinder und Jugendliche mit Behinderungen. Allein für Minderjährige, deren „leibliche, geistige oder seelische Entwicklung gefährdet oder geschädigt" war, sah §55 JWG die Bestellung eines Erziehungsbeistands und $\S 62 \mathrm{JWG}$ die Gewährung freiwilliger Erziehungshilfe vor, bei Gefahr im Verzug konnte das Vormundschaftsgericht gem. $§ 67$ Abs. 1 JWG die vorläufige Fürsorgeerziehung anordnen. Störungen in der seelischen Entwicklung wurden jugendhilferechtlich somit als Erziehungsdefizit behandelt. ${ }^{73}$

Ein Diskussionsentwurf aus dem Jahre 1973 zu einem Jugendhilfegesetz (JHG) forderte die Überführung junger Menschen mit seelischer Behinderung in die Jugendhilfe und ihre dortige Verortung im Rahmen der Erziehungshilfen, um Zuständigkeitsstreitigkeiten zwischen Jugend- und Sozialhilfe zu unterbinden, die aufgrund der Abgren-

69 Begründung der Bundesregierung zum „Entwurf eines Gesetzes zur Einordnung des Sozialhilferechts in das Sozialgesetzbuch“, BT-Drucks. 15/1514, S. 67.

70 Bayerisches LSG, Urt. v. 31.7.2006 - L 11 SO 27/06 - Rdnr. 22 (zitiert nach juris); Dietrich Schoch, in: LPK-SGB XII (Fußn. 14), §97 Rdnr. 12; vgl. auch: Volker Schlette, in: Karl Hauck/Wolfgang Noftz (Begr.), SGB XII, 2. Bd., 28. Lieferung, Stand: Juli 2012, Berlin 2012, § 97 Rdnr. 17, der $\S 97$ Abs. 2 SGB XII als „Blankettermächtigung des Landesgesetzgebers“ bezeichnet; ebenso: BVerwG, Beschl. v. 2.6.2008 - 5 B 188/07 - Rdnr. 6 (zitiert nach juris).

71 BGBl. I 1969, S. 1153.

72 BGBl. I 1961, S. 1206.

73 Clarita Schwengers, Eingliederungshilfen für seelisch behinderte Kinder und Jugendliche nach § 35a SGB VIII im Verhältnis zu konkurrierenden Leistungen nach dem (Sozial-)Leistungsrecht Zugleich ein Beitrag zu öffentlich-rechtlichen Ausgleichsansprüchen bei Doppelzuständigkeiten von Leistungsträgern, Stuttgart 2007, S. 43. 
zungsschwierigkeiten von seelischen Behinderungen und den Erziehungs- und Verhaltensstörungen entstanden. ${ }^{74}$ Dieser Diskussionsentwurf wurde nicht umgesetzt. ${ }^{75}$ Mit dem Gesetz zur Neuordnung des Kinder- und Jugendhilferechts (Kinder- und Jugendhilfegesetz - KJHG) vom 26. Juni 199076, gem. Art. 24 Satz 1 in Kraft getreten am 1. Januar 1991, wurden dann aber Maßnahmen der Eingliederungshilfe nach Maßgabe des $\S 40$ BSHG als Hilfen zur Erziehung in $\S 27$ Abs. 4 geregelt. ${ }^{77}$ Die Kollisionsnorm des $\S 10$ Abs. 2 Sätze 1 und 2 (jetzt $\S 10$ Abs. 4 Sätze 1 und 2) statuierte den Vorrang des SGB VIII für Maßnahmen der Eingliederungshilfe für junge Menschen mit seelischer Behinderung vor dem BSHG. Danach waren zwar die Schwierigkeiten bei der Abgrenzung von seelischer Behinderung, die zuvor der Zuständigkeit der Sozialhilfeträger unterlegen hatte, und einem jugendhilferechtlich zu deckenden erzieherischen Bedarf behoben, allerdings entzündete sich der Streit nunmehr an der Frage, ob Eingliederungshilfe für junge Menschen mit seelischer Behinderung als Bestandteil der Erziehungshilfen auch gewährt werden konnte, wenn ein Erziehungsdefizit i. S. d. § 27 Abs. 1 SGB VIII als Grundnorm für den Rechtsanspruch auf Hilfen zur Erziehung 78 nicht vorlag; andernfalls wären die Sozialhilfeträger bei Nichterweislichkeit eines Erziehungsdefizits immer auch für Maßnahmen der Eingliederungshilfe bei seelischer Behinderung von Kindern und Jugendlichen zuständig gewesen. ${ }^{79}$

Diesem neuerlich sich abzeichnenden Abgrenzungs- und demgemäß Zuständigkeitsproblem abzuhelfen, war schließlich die Einführung des § 35a SGB VIII als eigenständige Anspruchsnorm für Leistungen der Eingliederungshilfe bei seelischer Behinderung durch das Erste Gesetz zur Änderung des Achten Buches Sozialgesetzbuch vom 16. Februar 1993 (Art. 1 Nr. 17) ${ }^{80}$, gem. Art. 7 Abs. 1 Satz 2 des Ersten Änderungsgesetzes in Kraft getreten am 1. April 1993, zu dienen bestimmt. ${ }^{81}$

(2) Die Gesetzessystematik

Dass eine gesetzliche Verschiedenbehandlung von Kindern und Jugendlichen mit Behinderungen abhängig von der Behinderungsart nicht notwendig ist, um behinderungsbedingten Besonderheiten Rechnung zu tragen, belegt bereits der Umstand, dass $\S 35 a$ Abs. 3 SGB VIII im Hinblick auf Aufgaben, Ziele und Art der Eingliederungshil-

74 Bundesministerium für Jugend, Familie und Gesundheit (Hrsg.), Diskussionsentwurf eines Jugendhilfegesetzes, Bonn 1973, S. 121 (zu § 42), S. 122 f. (zu § 42 Absatz 1), S. 128 (zu § 47 Absatz 1, Fußn. 1).

75 Schwengers (Fußn. 73), S. 44.

76 BGBl. I 1990, S. 1163.

77 Dazu die Begründung der Bundesregierung zum „Entwurf eines Gesetzes zur Neuordnung des Kinder- und Jugendhilferechts (Kinder- und Jugendhilfegesetz - KJHG)“, BT-Drucks. 11/5948, S. 53.

78 Britta Tammen/Thomas Trenczek, in: Münder/Meysen/Trenczek (Fußn. 15), § 27 Rdnr. 1; Heike Schmid-Obkirchner, in: Wiesner (Fußn. 15), § 27 Rdnr. 1.

79 Siehe: Schwengers (Fußn. 73), S. 45.

80 BGBl. I 1993, S. 239.

81 Ausführlich dazu und zu den Debatten im Kontext mit § 35a SGB VIII: Schwengers (Fußn. 73), S. 45 ff. 
feleistungen keine eigenständigen Vorgaben trifft, sondern auf diejenigen des SGB XII, konkret auf $\S 53$ Abs. 3 und 4 Satz 1, §§ 54, 56 und 57 SGB XII, verweist. Dies macht im Gegenteil deutlich, dass der Gesetzgeber die differierenden leistungsrechtlichen und Zuständigkeitsnormen in einen möglichst einheitlichen Rahmen einpassen wollte, was das kompetenzrechtliche Moment als Ursache für den Dualismus ${ }^{82}$ umso stärker in den Vordergrund treten lässt.

\section{cc) Ergebnis}

Die Zuteilung von Kindern und Jugendlichen mit Behinderungen zu zwei unterschiedlichen Sozialleistungssystemen ist demnach nicht aufgrund behinderungsbezogener Besonderheiten unerlässlich, vielmehr ist die ,gesetzliche Segregation“ ${ }^{83}$ das Ergebnis von Zuständigkeitskonflikten zwischen den Trägern der öffentlichen Jugendhilfe und der Sozialhilfe und werden die praktischen wie rechtlichen Vorzüge einer einheitlichen Lösung von keiner Seite bestritten. Die rechtlich relevante Ungleichbehandlung ist daher nicht verfassungsrechtlich gerechtfertigt. ${ }^{84}$

\section{Auflösung der Ungleichbehandlung}

Ein Verstoß des Gesetzgebers gegen Art. 3 GG „kann [...] regelmäßig auf verschiedene Weise geheilt werden [...].“85 Welchen Weg der Gesetzgeber hier wählt, ,muss grundsätzlich der Gestaltungsfreiheit des Gesetzgebers überlassen bleiben [...].“86

Im Folgenden sollen die zwei vorliegend im Wesentlichen ins Feld geführten Lösungsmodelle, eine einheitliche Regelung der Eingliederungshilfe für Kinder und Jugendliche mit Behinderungen im SGB XII einerseits und im SGB VIII andererseits (,große Lösung“" $)^{87}$, unter dem Aspekt ihrer jeweiligen Vorzüge betrachtet werden.

Die Vorteile, die eine einheitliche Regelung ungeachtet des Regelungsortes mit sich bringt, werden der Bewertung der einzelnen Modelle vorangestellt.

82 Siehe unter II.2.c).

83 Thomas Meysen, Kinder- und Jugendhilfe an allen Schnittstellen: zentrale Anlaufstelle, Ausfallbürge, Netzeknüpfer, JAmt/ZKJ Sonderheft 2012, S. 21.

84 Siehe auch die Andeutung bei: Schwengers (Fußn. 73), S. 340.

85 BVerfGE 22, 349, 361; vgl. auch: BVerfGE 103, 225, 240; ferner: Pieroth/Schlink (Fußn. 41), Rdnr. 515; Jarass, in: ders./Pieroth (Fußn. 16), Art. 3 Rdnr. 40.

86 BVerfGE 22, 349, 361.

87 Zwischenbericht 2011 (Fußn. 4), S. 24 ff. 


\section{a) Vorzüge einer einheitlichen Regelung per se}

Die Vorzüge einer einheitlichen Regelung ergeben sich schon logisch aus den die rechtlich relevante Ungleichbehandlung begründenden und dementsprechend nachteilig wirkenden Divergenzen im Bereich des Leistungsrechts und der Zuständigkeit. ${ }^{88}$

Zum einen würde dadurch die Abhängigkeit eines Eingliederungshilfeanspruchs von unterschiedlichen Leistungsvoraussetzungen beseitigt, zum anderen könnten die Leistungen im Falle von Mehrfachbehinderungen aus einer Hand erbracht und auf Zuständigkeitsstreitigkeiten basierende Leistungsverzögerungen, insbesondere bei Schwierigkeiten der Zuordnung der Beeinträchtigung zu einer Behinderungsart ${ }^{89}$, vermieden werden. Zwar verpflichtet $\S 14$ Abs. 1 Satz 1 SGB IX ${ }^{90}$ den angegangenen Rehabilitationsträger, innerhalb von zwei Wochen nach Eingang des Antrags bei ihm festzustellen, „ob er nach dem für ihn geltenden Leistungsgesetz für die Leistung zuständig ist [...].“91 Die Regelung soll ,durch rasche Klärung von Zuständigkeiten Nachteilen des gegliederten Systems entgegen[zu]wirken.“92 Allerdings wäre dies bei einer einheitlichen Regelung überhaupt nicht erforderlich.

Hinzu kommt, dass im Falle der gerichtlichen Auseinandersetzung ein einheitlicher Rechtsweg zur Verfügung stünde. 93

\section{b) Vorzüge einer einheitlichen Regelung im SGB XII}

Für die einheitliche Regelung der Eingliederungshilfe für Kinder und Jugendliche mit Behinderungen im SGB XII wird im Wesentlichen die Vermeidung eines Zuständigkeitswechsels bei einem Übertritt in die Volljährigkeit angeführt, wenn also die Phase „Kindheit und Jugend“ i. S. d. $§ 7$ Abs. 1 Nrn. 1 und 2 SGB VIII endet. ${ }^{94}$ Für junge

88 Siehe oben II.2.b).

89 Ein Beispiel ist Autismus: Helmut Remschmidt/Christian Frese, Aktuelle Entwicklungen bei der sozialrechtlichen Zuordnung autistischer Störungen - $\mathrm{Zu}$ den Folgen des Urteils des OVG Nordrhein-Westfalen vom 20.2.2002, Az: 12 A 5322/00, SGb 2006, S. 410 ff.; Reinhart Lempp, Die seelische Behinderung bei Kindern und Jugendlichen als Aufgabe der Jugendhilfe, 5. Aufl. Stuttgart 2006, S. 38 ff.; Schwengers (Fußn. 73), S. 147 ff.; Franz Dillmann/Knut-Egbert Dannat, „Forever young“ - Ewig junge Abgrenzungsprobleme zwischen Leistungen für junge behinderte Menschen nach dem SGB VIII und dem SGB XII, ZfF 2009, S. 25, 26; Fegert, in: Wiesner (Fußn. 15), § 35a Rdnr. 75 ff.; BT-Drucks. 16/12860, S. 13.

90 Dazu, dass dieser gem. § 7 Satz 1 Halbsatz 1 SGB IX mangels abweichender Regelungen auch im Zusammenhang mit $\S 10$ SGB VIII Anwendung findet: Meysen, in: Münder/ders./Trenczek (Fußn. 15), § 10 Rdnr. 47 und § 35a Rdnr. 81 ff.; Wiesner, in: ders. (Fußn. 15), § 10 Rdnr. 49; Wel$t i$, in: HK-SGB IX (Fußn. 14), § 14 Rdnr. 2.

91 Ausführlich zu dem Procedere: Welti, in: HK-SGB IX (Fußn. 14), § 14 Rdnr. 34 ff.; Peter Ulrich, Die (Nicht-)Weiterleitung des Teilhabeantrages und ihre Folgen - §14 SGB IX als gesetzesübergreifende Nahtstelle materiell- und verfassungsrechtlicher Fragen, SGb 2008, S. 452, 454 ff., 456 ff.

92 BT-Drucks. 14/5074, S. 102.

93 Zum Ganzen auch: Zwischenbericht 2011 (Fußn. 4), S. 24 ff.

94 Abwägend dazu: Greß/Rixen/Wasem (Fußn. 7), VSSR 2009, S. 43, 53 ff. 
Volljährige, mithin Personen, die das 18., aber noch nicht das 27. Lebensjahr vollendet haben (§ 7 Abs. 1 Nr. 3 SGB VIII) und die nach Maßgabe des $§ 41$ SGB VIII unter Umständen noch Eingliederungshilfe nach $\S 35$ a SGB VIII beanspruchen können, würde die Gefahr der Entstehung von Doppelstrukturen vermieden. ${ }^{95}$

Außerdem sprenge § 35a SGB VIII „,die Logik, Systematik und die Strukturmaximen des SGB VIII“, weil dieses von seinem Selbstverständnis her ein Erziehungsgesetz sei. ${ }^{96}$ Darüber hinaus werde mit $\S 35$ a SGB VIII allein für die Gruppe der seelisch behinderten jungen Menschen ein individueller Rechtsanspruch eröffnet, während im Übrigen SGB VIII - dem Gesamtkonzept des Gesetzes entsprechend - immer die Eltern beziehungsweise die Personensorgeberechtigten leistungsberechtigt seien. 97

\section{c) Vorzüge einer einheitlichen Regelung im SGB VIII (,,große Lösung “)}

Für eine Zusammenfassung der Eingliederungshilfe für Kinder und Jugendliche mit Behinderungen unter dem Dach des SGB VIII spricht zum einen die gesetzessystematische Erwägung, dass die Verankerung der Eingliederungshilfe im SGB XII an sich nicht konsistent ist. Hinzu kommt die größere Sachnähe des SGB VIII.

\section{aa) Die gesetzliche Verortung der Eingliederungshilfe im SGB XII ${ }^{98}$}

Grundgesetzlicher Kompetenztitel für die Sozialhilfe ist Art. 74 Abs. 1 Nr. 7, der von „öffentlicher Fürsorge“ spricht. Darunter ist grundsätzlich die behördliche Unterstützung Hilfebedürftiger zu verstehen, die sich in einer individuellen Notlage befinden. ${ }^{99}$ Die Sozialhilfe als „das letzte Netz der sozialen Sicherung"100 ist daher der Prototyp öffentlicher Fürsorge. Ihre Aufgabe ist es gem. § 1 Satz 1 SGB XII, „den Leistungsberechtigten die Führung eines Lebens zu ermöglichen, das der Würde des Menschen entspricht" (vgl. auch § 9 SGB I).

Besondere Aufgabe der Eingliederungshilfe ist es hingegen gem. § 53 Abs. 3 Satz 1 SGB XII, „eine drohende Behinderung zu verhüten oder eine Behinderung oder deren Folgen zu beseitigen oder zu mildern 101 und die behinderten Menschen in die Gesellschaft einzugliedern.“ Hierzu gehört nach Satz 2 insbesondere, den behinderten Men-

95 Zwischenbericht 2011 (Fußn. 4), S. 26.

96 So die Begründung der Bundesregierung zum „Entwurf eines Gesetzes zur Entlastung der Kommunen im sozialen Bereich (KEG)“, BT-Drucks. 15/4532, S. 14.

97 BT-Drucks. 15/4532, S. 14.

98 Siehe bereits: Banafsche (Fußn. 13), SGb 2012, S. 373, 376 ff.

99 Peter Lerche, Verfassungsfragen um Sozialhilfe und Jugendwohlfahrt - Ein Rechtsgutachten, Berlin 1963, S. 16, 44; ferner: Christoph Degenhart, in: Sachs (Fußn. 28), Art. 74 Rdnr. 35; Stefan Oeter, in: von Mangoldt/Klein/Starck (Fußn. 17), Art. 74 Rdnr. 55; Rupert Stettner, in: Horst Dreier (Hrsg.), Grundgesetz, Bd. II, 2. Aufl. Tübingen 2006, Art. 74 Rdnr. 44.

100 Gerhard Igl/Felix Welti, Sozialrecht, 8. Aufl. Neuwied 2007, § 55 Rdnr. 1.

101 Nähere Ausgestaltung durch die Leistungen zur medizinischen Rehabilitation gem. § 54 Abs. 1 Satz 1 SGB XII i. V. m. $\S \S 26$ bis 32 SGB IX. 
schen die Teilnahme102 am Leben in der Gemeinschaft zu ermöglichen oder zu erleichtern ${ }^{103}$, ihnen die Ausübung eines angemessenen Berufs oder einer sonstigen angemessenen Tätigkeit zu ermöglichen ${ }^{104}$ oder sie so weit wie möglich unabhängig von Pflege zu machen. ${ }^{105}$ Es geht also bei behinderungsbedingten Leistungen - nicht zuletzt angesichts der Entwicklungen der vergangenen 20 Jahre, beginnend mit der Einfügung des Art. 3 Abs. 3 Satz 2 in das Grundgesetz durch das Gesetz zur Änderung des Grundgesetzes vom 27. Oktober 1994106 - weniger um Fürsorge als vielmehr um einen Ausgleich gesellschaftlicher beziehungsweise umweltbedingter Nachteile. ${ }^{107}$ Diese Entwicklung weg vom Fürsorgedenken und hin zu einer menschenrechtlichen Betrachtungsweise von behinderungsbedingt zu gewährenden Leistungen hat in der UN-BRK mit ihren Paradigmata der Selbstbestimmung und Teilhabe (Art. 3 Buchstaben a und c), die bereits dem SGB IX zugrunde liegen (vgl. § 1) und in Wechselwirkung zueinander stehen ${ }^{108}$, abermals einen starken Ausdruck gefunden. 109

Schließlich geht auch das durch $\S 53$ Abs. 3 Satz 2 SGB XII bereits angedeutete Leistungsspektrum der Eingliederungshilfe, wie es die $\S \S 54$ bis 56 SGB XII i. V. m. $\S \S 6$ bis 24 EinglH-VO abbilden, in seiner Breite über die nach den übrigen teilhaberelevanten Leistungsgesetzen $110 \mathrm{zu}$ gewährenden Hilfen hinaus und entfaltet insoweit eine eigenständige Bedeutung ${ }^{111}$, der eine Zuordnung zu der Sozialhilfe nicht gerecht zu werden vermag. ${ }^{112}$

102 Teilnahme setzt demgemäß die Möglichkeit zur Teilhabe voraus, so dass Teilhabeschranken zu Teilnahmehindernissen führen. Zu dem Verhältnis von Teilhabe und Teilnahme auch: Klaus Lachwitz, in: HK-SGB IX (Fußn. 14), Anhang 2 Rdnr. 5.

103 Nähere Ausgestaltung durch die Leistungen zur Teilhabe am Leben in der Gemeinschaft gem. §54 Abs. 1 Satz 1 SGB XII i. V. m. $\S \S 55$ bis 59 SGB IX.

104 Nähere Ausgestaltung durch die Leistungen zur Teilhabe am Arbeitsleben gem. § 54 Abs. 1 Satz 1 SGB XII i. V. m. $\S \S 33$ bis 43 SGB IX.

105 Dies entspricht der Zielsetzung des SGB IX, die Selbstbestimmung von Menschen mit Behinderungen sowie ihre ,gleichberechtigte Teilhabe am Leben in der Gesellschaft zu fördern, Benachteiligungen zu vermeiden oder ihnen entgegenzuwirken“ ( 1 Satz 1), an welcher sich die Leistungen zur Teilhabe nach Maßgabe des $\S 4$ Abs. 1 SGB IX ausrichten.

106 BGBl. I 1994, S. 3146.

107 Antje Welke, Die Zukunft der Eingliederungshilfe für Menschen mit Behinderung, NDV 2009, S. 456, 457; Wahrendorf, in: Grube/ders. (Fußn. 14), § 53 Rdnr. 35.

108 Für die UN-BRK: Heiner Bielefeldt, Zum Innovationspotenzial der UN-Behindertenrechtskonvention, Essay No. 5, 3. Aufl. Berlin 2009, S. 10 f.; stellvertretend für das SGB IX: Welti, in: HKSGB IX (Fußn. 14), § 1 Rdnr. 12, 14.

109 Dazu auch: Stefan Kurzke-Maasmeier, Von der Fürsorge zur Selbstbestimmung - Die UNBehindertenrechtskonvention als Herausforderung für Soziale Dienste, Soziale Professionen und Gemeinwesen, S. 1 ff., abrufbar unter: http://www.imew.de/fileadmin/Dokumente/Volltexte/ Tagungen_2009/UN-Konvention_25.06.09/Kurzke-Maasmeier.htm (letzter Zugriff: 18.03.2012).

110 Ausgehend von den $\S \S 6$ und 6a SGB IX sind dies das SGB V, das SGB III, das SGB VII, das SGB VI und das ALG, das BVG, das SGB VIII, das SGB XII und das SGB II.

111 Die eigenständige Bedeutung der Eingliederungshilfe nach dem SGB XII insbesondere hinsichtlich der Leistungen zur Teilhabe am Leben in der Gemeinschaft herausstellend: Felix Welti, Systematische Stellung des SGB IX im Sozialgesetzbuch - Zusammenarbeit der Leistungsträger und Koordi- 
Bei dem Einwand, § 35a SGB VIII füge sich nicht in die Systematik des SGB VIII ein 113 , wird neben der dargelegten systematischen Inkonsistenz von Eingliederungshilfe und Sozialhilfe in der Sache nicht hinreichend berücksichtigt, dass das SGB VIII nicht nur die Erziehung junger Menschen, sondern gleichermaßen ihre Entwicklung fokussiert. 114 Dabei geht es gem. § 1 Abs. 3 Nr. 1 SGB VIII um die individuelle und soziale Entwicklung, die es zu fördern und im Rahmen derer es Benachteiligungen zu vermeiden oder abzubauen gilt, was mit den Zielen der Eingliederungshilfe nach dem oben Gesagten gerade übereinstimmt.

Auch die Anspruchsberechtigung der Kinder und Jugendlichen selbst durch $\S 35 \mathrm{a}$ Abs. 1 Satz 1 SGB VIII steht dem Gesamtkonzept des SGB VIII nicht entgegen. 115 Dies belegen im Besonderen die Anspruchsleistungen nach $\S 13$ Abs. 1 SGB VIII auf sozialpädagogische Hilfen ${ }^{116}$, nach $\S 18$ Abs. 3 Satz 1 SGB VIII auf Beratung und Unterstützung bei der Ausübung des Umgangsrechts nach § 1684 Abs. 1 BGB, nach § 24 Abs. 1 Satz 1 SGB VIII auf Besuch einer Tageseinrichtung sowie nach $\S 41$ Abs. 1 Satz 1 SGB VIII auf Hilfe für junge Volljährige. Dass eine leistungsrechtliche Adressierung junger Menschen als Anspruchsberechtigte im Gesamtkonzept des SGB VIII im Gegenteil sogar angelegt ist, macht auch die allgemeine Vorschrift des $\S 8$ SGB VIII deutlich, die für die Wahrnehmung aller Aufgaben der Jugendhilfe - gem. § 2 SGB VIII sind dies Leistungen und andere Aufgaben - gilt ${ }^{117}$ und in Absatz 3 Satz 1 unter den dort genannten Voraussetzungen einen Anspruch für Kinder und Jugendliche auf Beratung ohne Kenntnis der Personensorgeberechtigten i. S. d. $§ 7$ Abs. 1 Nr. 5 SGB VIII statuiert; dass nun eine übergeordnete Norm ihrerseits Vorgaben enthalten soll, die dem Gesamtkonzept des SGB VIII zuwiderlaufen, erhellte nicht.

nierung der Leistungen, SGb 2008, S. 321, 328; Felix Welti, Die Rehabilitation im System des Sozialleistungsrechts aus rechtswissenschaftlicher Sicht, in: ders. (Hrsg.), Das Rehabilitationsrecht in der Praxis der Sozialleistungsträger, Berlin 2009, S. 16, 28.

112 Dass es sich bei der Eingliederungshilfe um eine sozialleistungsübergreifende Hilfe handelt, lässt sich auch an ihrem Verhältnis zum SGB II festmachen: Banafsche (Fußn. 13), SGb 2012, S. 373, 377.

113 BT-Drucks. 15/4532, S. 14, siehe oben B.III.2.

114 Von einem vollständigen Übersehen kann wohl nicht ausgegangen werden, weil die Entwurfsbegründung zumindest wie folgt ausführt: „Ansatz des SGB VIII ist, die Entstehung und Lösung von Problemen des Aufwachsens, der Erziehung und der Entwicklung junger Menschen in ihrer wechselseitigen Abhängigkeit als multifaktorielle Erziehungs- und Entwicklungsbedingungen zu sehen“, BT-Drucks. 15/4532, S. 14.

115 So aber: BT-Drucks. 15/4532, S. 14, siehe oben II.3.b).

$116 \mathrm{Ob}$ es sich bei $\S 13$ Abs. 1 SGB VIII lediglich um eine objektive Gewährleistungspflicht des Trägers der öffentlichen Jugendhilfe handelt, wie es der Normwortlaut auf den ersten Blick nahelegt (,jungen Menschen [...] sollen [...] angeboten werden“), oder um ein subjektiv-öffentliches Recht, weil es um die „Überwindung individueller Beeinträchtigungen“ geht, ist umstritten. Mit weiteren Nachweisen dazu: Jutta Struck, in: Wiesner (Fußn. 15), § 13 Rdnr. 7.

117 Meysen, in: Münder/ders./Trenczek (Fußn. 15), § 8 Rdnr. 1. 


\section{bb) Größere Sachnähe des SGB VIII}

Legt man nach dem Gesagten einerseits zugrunde, dass behinderungsbedingte Leistungen nicht (mehr) als Fürsorgeleistungen, sondern als Ausgleich für gesellschaftliche beziehungsweise umweltbedingte Nachteile zu begreifen sind ${ }^{118}$, und berücksichtigt andererseits, dass Leistungen der sozialen Förderung - wie der Kinder- und Jugendhilfe nach dem SGB VIII - gerade auf die Herstellung sozialer Chancengleichheit und ,zivilisatorischer Teilhabe [...] über das Existenzminimum hinaus“" zielen, während die soziale Hilfe - wie die Sozialhilfe nach dem SGB XII - allein der Absicherung des Existenzminimums im Sinne einer der Würde des Menschen entsprechenden Lebensführung (vgl. § 1 Satz 1 SGB XII und § 1 Abs. 1 SGB II jeweils i. V. m. Art. 1 Abs. 1, Art. 20 Abs. $1 \mathrm{GG}^{119}$ ) dient ${ }^{120}$, kommt man im Hinblick auf Eingliederungshilfeleistungen für Kinder und Jugendliche nicht umhin, dem SGB VIII die größere Sachnähe beizumessen. ${ }^{121}$ Zum gleichen Ergebnis gelangt auch der 13. Kinder- und Jugendbericht, der die Leistungen für Kinder und Jugendliche mit Behinderungen an der Lebenslage „Kindheit und Jugend" und nicht an dem Vorliegen einer Behinderung ausgerichtet wissen will. 122

Die Jugendämter wiederum orientieren sich bei der Durchführung der Leistungen an den allein auf die Bedürfnisse der Kinder und Jugendlichen sowie Familien insgesamt zugeschnittenen Grundsätzen der Kinder- und Jugendhilfe (vgl. § 1 Abs. 3 SGB VIII als „Generalklausel und Leitnorm“123). Die Pflicht zur Einbeziehung der Jugendämter bei der Aufstellung eines Gesamtplans zur Durchführung der einzelnen Leistungen gem. $\S 58$ SGB XII ${ }^{124}$ vermag die originär jugendhilferechtliche Hilfeplanung nach $\S 36$ Abs. 2 bis 4 SGB VIII nicht zu ersetzen, der in Absatz 2 Satz 2, die Ausgestaltung der Hilfe betreffend, entscheidende Maßgaben festlegt. 125

118 Siehe oben II.3.c)aa).

$119 \mathrm{Zu}$ dem Grundrecht auf Gewährleistung eines menschenwürdigen Existenzminimums aus Art. 1 Abs. 1 i. V. m. Art 20 Abs. 1 GG: BVerfGE 40, 121, 133; 45, 187, 228; 82, 60, 85; 113, 88, 108 f;; 123, 267, 362 f.; 125, 175, 222 ff.; für die Literatur: Starck, in: von Mangoldt/Klein/ders. (Fußn. 19), Art. 1 Rdnr. 41.

120 Hans Friedrich Zacher, Entwicklung einer Dogmatik des Sozialrechts, in: Maximilian Wallerath (Hrsg.), Fiat iustitia - Recht als Aufgabe der Vernunft - Festschrift für Peter Krause zum 70. Geburtstag, Berlin 2006, S. 3, 11; in der Sache auch: Felix Welti, Das Recht auf Teilhabe behinderter Menschen in seinem verfassungs- und europarechtlichen Rahmen und im Spannungsfeld der neueren Sozialgesetzgebung, RsDE 62 (2006), S. 1, 15.

121 Vgl. bereits in der Sache: BT-DruckS. 11/5948, S. 53.

122 BT-Drucks. 16/12860, S. 12.

123 BT-Drucks. 11/5948, S. 47.

124 Vgl.: Bieritz-Harder, in: LPK-SGB XII (Fußn. 14), § 58 Rdnr. 3.

125 Der Rechtscharakter des $\S 36$ Abs. 2 Satz 2 SGB VIII wird nicht einheitlich bewertet: Von einem „subjektiv-rechtlichen Anspruch auf weitere qualifizierte Beteiligung an der Entscheidungsfindung im Hilfeplanprozess“ spricht: Meysen, in: Münder/ders./Trenczek (Fußn. 15), § 36 Rdnr. 22; dagegen für ein Zugestehen ,rein jugendamtsinterne[r] und ,verfahrensdienende[r] ${ }^{`}$ Aufgaben und Funktionen“: Reinhard Joachim Wabnitz, Rechtsansprüche gegenüber Trägern der öffentlichen Kinder- 


\section{d) Ergebnis}

Aus den genannten gesetzessystematischen Erwägungen sowie aufgrund der größeren Sachnähe des SGB VIII nicht nur zu der Lebenslage „Kindheit und Jugend“, sondern auch zu dem Ziel der Eingliederungshilfe, behinderungsbedingte Nachteile auszugleichen und auf diese Weise soziale Chancengleichheit und gesellschaftliche Teilhabe zu generieren, ist eine einheitliche Regelung der Eingliederungshilfe für Kinder und Jugendliche mit Behinderungen im SGB VIII, mithin die „große Lösung“, zu präferieren. 126

\section{Schluss}

An das unter B.III. gefundene Ergebnis anknüpfend, soll nun erneut auf den eingangs zitierten Ausschnitt aus dem 13. Kinder- und Jugendbericht rekurriert werden, der die Lebenslage „Kindheit und Jugend“ in den Vordergrund rückt und die „Behinderung“ als sekundäres Moment wertet. 127

Gleiches ergibt sich bereits aus der Stellungnahme des Bundesrates zu dem „Entwurf eines Sozialgesetzbuchs - Neuntes Buch - (SGB IX) Rehabilitation und Teilhabe behinderter Menschen“, konkret zu § 4 Abs. 3 SGB IX (§ 1 Abs. 2 der Entwurfsfassung), wonach „Leistungen für behinderte oder von Behinderung bedrohte Kinder [...] so geplant und gestaltet [werden], dass nach Möglichkeit Kinder nicht von ihrem sozialen Umfeld getrennt und gemeinsam mit nicht behinderten Kindern betreut werden können. Dabei werden behinderte Kinder alters- und entwicklungsentsprechend an der Planung und Ausgestaltung der einzelnen Hilfen beteiligt und ihre Sorgeberechtigten intensiv in Planung und Gestaltung der Hilfen einbezogen." In besagter Stellungnahme heißt es: „Die Standards des SGB VIII sollten als Gestaltungsauftrag auch für das SGB IX genannt werden." 128

Das SGB IX schafft damit in Bezug auf Kinder und Jugendliche mit Behinderungen selbst die Anbindung an die Maßgaben des SGB VIII, ohne zwischen Behinderungsarten zu unterscheiden. In Anbetracht dessen ist die größere Sachnähe des SGB VIII zu

und Jugendhilfe nach dem Achten Buch Sozialgesetzbuch (SGB VIII), Berlin 2005, S. 210 f.; siehe auch: BT-Drucks. 11/5948, S. 74, wonach der Hilfeplan in erster Linie als „Instrument der Selbstkontrolle für das verantwortliche Jugendamt“ sowie als „Koordinierungsinstrument zwischen dem Jugendamt und dem Träger der Einrichtung, der im Einzelfall tätig wird“ dient.

126 Mit weiteren Nachweisen für die „große Lösung“: Banafsche (Fußn. 6), S. 116, 123, Fußn. 100; jüngst auch der Bericht über die Lebenssituation junger Menschen und die Leistungen der Kinderund Jugendhilfe in Deutschland - 14. Kinder- und Jugendbericht, BT-Drucks. 17/12200, S. 17, $370 f$.

127 BT-Drucks. 16/12860, S. 12, siehe unter I. 
eben dieser Lebenslage „Kindheit und Jugend“ ein wichtiges Argument für die „große Lösung“".

Schließlich wird auch die zentrale Leitidee des Art. 6 Abs. 2 GG, wonach Pflege und Erziehung der Kinder das natürliche Recht der Eltern und die ihnen zuvörderst obliegende Pflicht sind, über deren Betätigung die staatliche Gemeinschaft wacht - das Kindeswohl als bei allen Kinder und Jugendliche betreffenden Maßnahmen vorrangig zu berücksichtigender Gesichtspunkt (vgl. Art. 3 Abs. 1 UN-Kinderrechtskonvention (UNKRK) und Art. 7 Abs. 2 UN-BRK) - durch das SGB VIII, welches Art. 6 Abs. 2 GG in $\S 1$ Abs. 2 wortlautidentisch wiederholt und damit in seinen Handlungsauftrag 129 einbezieht, maßgeblich ausgestaltet ${ }^{130}$ und wird somit die „große Lösung“ zum verfassungsrechtlichen Gebot.

Abschließend sei daher der Rekurs auf den Philosophen David Hume gestattet, der 1779 schrieb: „Zahllose Universen mögen während einer Unendlichkeit verpfuscht und vermasselt worden sein, bevor dieses System sich durchsetzte; viel vergebliche Arbeit, viele erfolglose Versuche und eine langsame, aber beständige Verbesserung, ausgeführt während unendlicher Zeitalter in der Kunst der Weltenschaffung."131

Dies alsbald sagen zu können, bedeutete nicht zuletzt im Sinne des Gleichheitsrechts des Art. 3 GG, dass die Debatte um die ,große Lösung“ nicht zu einer ,unendlichen Geschichte“ geworden wäre ${ }^{132}$, sondern zu einem positiven Ende gefunden hätte.

129 Vgl.: Münder, in: ders./Meysen/Trenczek(Fußn. 15), § 1 Rdnr. 1 f.

130 Zur Notwendigkeit der gesetzlichen Ausgestaltung des Elternrechts des Art. 6 Abs. 2 GG: BVerfGE 84, 168, 180; ferner: Hans Friedrich Zacher, Elternrecht, in: Josef Isensee/Paul Kirchhof (Hrsg.), Handbuch des Staatsrechts der Bundesrepublik Deutschland, Bd. VI, Heidelberg 1989, § 134 Rdnr. 48; Jarass, in ders./Pieroth (Fußn. 16), Art. 6 Rdnr. 51.

131 Zitiert nach: Marcus Chown, Das Universum nebenan - Revolutionäre Ideen in der Astrophysik, Köln 2003, S. 161.

132 Siehe den Beitrag von Wiesner, S. 153 in diesem Band. 


\title{
Kinder und Jugendliche mit Behinderungen in der Kommune - Kommentar aus Sicht eines Jugendamtes
}

\author{
Maria Kurz-Adam
}

I. Die „Große Lösung“ als Weg zur Inklusion für Kinder und Jugendliche in den Kommunen - Anmerkungen zum aktuellen Diskussionsstand in der Kinder- und Jugendhilfe

II. Inklusion in der Kinder- und Jugendhilfe - Widersprüche und Herausforderungen

III. Fazit: Perspektiven einer inklusiven Kinder- und Jugendhilfe - neue Hilfearchitekturen für eine inklusive „Hilfe zur Entwicklung“

I. Die „,Große Lösung“ als Weg zur Inklusion für Kinder und Jugendliche in den Kommunen - Anmerkungen zum aktuellen Diskussionsstand in der Kinder- und Jugendhilfe

Die Debatten und die Literatur zur „Großen Lösung“ - also der Zusammenführung der Zuständigkeit für die Eingliederungshilfe für geistig und körperlich behinderte Kinder und Jugendliche und für seelisch behinderte Kinder und Jugendliche im Leistungssystem der Kinder- und Jugendhilfe - sind mittlerweile fast Legion. Die wesentlichen vornehmlich fachlichen - Argumente für eine solche Zusammenführung liegen insbesondere seit dem Erscheinen des 11. Kinder- und Jugendhilfeberichts auf dem Tisch: eine Reduzierung der Schnittstellen zwischen Sozialrecht und Kinder- und Jugendhilferecht, eine damit verbundene „Verminderung der Abgrenzungs-, Zuständigkeits- und Konkurrenzprobleme“" insbesondere bei Mehrfachbehinderungen im Indikationsfeld zwischen geistiger und seelischer Behinderung sind die wesentlichen fachlichen Argumente des Berichts, der die getrennten Zuständigkeiten seit Inkrafttreten des § 35a SGB VIII letztlich für gescheitert hält. Das Zurechtfinden in den unterschiedlichen Zuständigkeiten der dualen Eingliederungshilfe für Kinder- und Jugendliche ist gerade aus Betroffenensicht, und besonders aus Sicht der hilfesuchenden Eltern, zu einer Aufgabe geworden, die ohne professionelle Beratung und Unterstützung kaum mehr zu schaffen ist: im Dickicht der Rechtsbestimmungen und Vorschriften scheint der Hilfeanspruch 